CERN-TH.6652/92

FTUV/92-35

IFIC/92-34

\title{
INVISIBLE HIGGS DECAYS AND NEUTRINO PHYSICS
}

\author{
Anjan S. Joshipura ${ }^{1 *}$ \\ Theoretical Physics Division, CERN \\ CH-1211 Geneve 23, Switzerland \\ and \\ J. W. F. Valle ${ }^{\dagger}$ \\ Instituto de Física Corpuscular - IFIC/CSIC \\ Dept. de Física Teórica, Universitat de Valencia \\ 46100 Burjassot, Valencia, SPAIN
}

\begin{abstract}
A wide class of neutrino physics-motivated models are characterized by the spontaneous violation of a global $U(1)$ lepton number symmetry at or below the electroweak scale by an $S U(2) \otimes U(1)$ singlet vacuum expectation value $\langle\sigma\rangle \lesssim \mathcal{O}(1) \mathrm{TeV}$. In all these models the main Higgs decay channel is likely to be "invisible", e.g. $h \rightarrow J J$, where $J$ denotes the associated weakly interacting pseudoscalar Goldstone boson the majoron. This leads to events with large missing energy that could be observable at LEP and affect the Higgs mass bounds obtained, as well as lead to novel ways to search for Higgs bosons at high energy supercolliders such as the LHC/SSC.
\end{abstract}

CERN-TH.6652/92

September 1992

${ }^{1}$ Permanent address: Theory Group, Physical Research Lab., Ahmedabad, India

\footnotetext{
* Bitnet JOSHIPUR@CERNVM

${ }^{\dagger}$ Bitnet VALLE@EVALUN11 - Decnet 16444::VALLE
} 


\section{Introduction}

One of the main puzzles in particle physics today is the problem of mass generation. It is believed that the masses of the fermions as well as that of gauge bosons arise as a result of the spontaneous breaking of the gauge symmetry. The key ingredient for this scenario, namely the Higgs boson [1], has not yet been found. It is only recently, with the LEP experiments, that one has seriously started constraining the relevant parameters, including the Higgs boson mass [2]. The limits on the Higgs mass are, however, rather model dependent. The present limit on the standard model Higgs coming from the data on $e^{+} e^{-}$collisions at LEP is $\sim 60 \mathrm{GeV}$.

An extension of the minimal standard model is desirable for many reasons. One is the question of neutrino masses. Indeed neutrino masses vanish in the minimal standard model and almost all attempts to induce them require an enlargement in the Higgs sector of the theory [3]. Among these, models known as majoron models are particularly interesting and have been extensively studied [3]. The majoron is a Goldstone boson associated with the spontaneous breaking of the lepton number. In the models we shall consider it has very tiny couplings to the charged fermions as well as to the gauge bosons. As a consequence, the majoron remains invisible.

The majoron can however have significant couplings to Higgs bosons even if its other couplings are suppressed. This could have important implications for Higgs physics. In particular, the normal doublet Higgs boson could decay invisibly as

$$
h \rightarrow J+J,
$$

where $J$ denotes the Goldstone boson -the majoron - associated with spontaneously broken lepton number symmetry. The possibility of a Higgs boson decaying invisibly was raised by Shrock and Suzuki and reconsidered by Li, Liu and Wolfenstein [4] in the context of the triplet majoron model [5]. This type of models are now excluded since they lead to an invisible $Z$ width in conflict with LEP observations [6]. Despite this, the possibility of invisible Higgs decay still remains open and experimentally very amusing [7]. A concrete example [8] was recently provided in the context of supersymmetric $S U(2) \otimes U(1)$ models where the $R$ parity is spontaneously violated at (or below) the electroweak scale [9]. The lightest Higgs boson $h$ decays in this model through majoron emission. Unfortunately, its production rates are likely to be small in this case, especially in the low mass region. While this completely avoids 
the existing LEP1 limits, it is not so useful for the experimental detection of the new effects at LEP1 (prospects of observing such decays are better at higher energies).

The above type of suppression in the production of the low mass Higgs boson need not occur in all models. An example where such suppression can be absent is provided [10] by the seesaw majoron model [11], provided the scale of lepton number violation obeys $\langle\sigma\rangle \lesssim \mathcal{O}(1) \mathrm{TeV}$. This model, with such vacuum expectation value (VEV), may have interesting physical implications including neutrinos with masses very near their present experimental limits [12]. However, this is not the most natural choice for the lepton number violation scale if neutrino masses are very tiny $\ll \mathcal{O}(1) \mathrm{eV}$. The masses of the light neutrinos are given by

$$
m_{\nu} \sim \frac{m_{D}^{2}}{M_{R}}
$$

where $m_{D}=\lambda\langle\phi\rangle$ and $M_{R} \sim\langle\sigma\rangle$. Here $\langle\phi\rangle$ is the VEV that breaks the $S U(2) \otimes U(1)$ symmetry while $\langle\sigma\rangle$ breaks the global lepton number symmetry. Barring unnaturally small Yukawa couplings $\lambda$, the smallness of neutrino masses follows only if $\langle\sigma\rangle \gg$ $\mathcal{O}(1)$ TeV. Typical models associate $\langle\sigma\rangle$ to a large mass scale at which some higher symmetry such as left-right, Peccei-Quinn or grand-unified symmetries get realized. As we shall discuss the majoron-Higgs coupling is suppressed in this case.

In this paper, we note that there exists a wide class of interesting models for neutrino masses in which lepton number breaking is driven by an isosinglet VEV (as required by the LEP constraints), but in which the associated scale obeys $\langle\sigma\rangle \lesssim \mathcal{O}(1) \mathrm{TeV}$. The distinguishing feature of these new models is that, unlike seesaw models, where $m_{\nu} \propto\langle\sigma\rangle^{-1}$, in any of the present models $m_{\nu} \rightarrow 0$ as $\langle\sigma\rangle \rightarrow 0$. As a result a low value of $\langle\sigma\rangle$ is required in order to obtain a small neutrino mass either at the tree level or radiatively.

We discuss the invisible decay of the Higgs bosons in this type of models. In contrast with the two situations discussed above, neither the invisible decay nor the production of the Higgs bosons need to be suppressed in these models. Moreover, this feature persists even when the lepton number symmetry is broken at a scale much smaller than the weak scale. The latter would lead to the possibility of enhanced majoron-neutrino couplings. These could, in turn, have interesting implications in neutrinoless double beta decay as well as astrophysics [13]. In addition, in all cases these models can lead to interesting physical effects such as large rates for zen events at LEP, and flavour-violating muon and tau decays with large branching ratios. The 
former would be associated to single neutral heavy lepton production and the latter to neutral heavy lepton exchange in higher order weak processes. The corresponding rates can be large enough to be experimentally measurable. [14].

In the next section, we discuss the main features of various models of neutrino masses with the lepton number broken at a relatively low scale. The third section contains details of the Higgs potentials and the majoron couplings to the Higgs bosons. The corresponding decay and production rates are studied in section 4 . The last section contains a discussion of some of the phenomenological implications. The technical details related to very low-scale breaking of the lepton number symmetry are given in the appendix.

\section{Models}

We now consider several $S U(2) \otimes U(1)$ models that have been suggested in neutrino physics in order to generate naturally small neutrino masses, either as a result of radiative corrections or at the tree level ${ }^{\ddagger}$. In all these models lepton number is a symmetry of the Lagrangian. This is spontaneously broken by $\langle\sigma\rangle \lesssim \mathcal{O}(1) \mathrm{TeV}$, thus generating a majoron given by

$$
J=\operatorname{Im} \sigma
$$

In most respects, these models all share the existence of a massless isosinglet pseudoscalar majoron, very much the same as the original one in ref. [11]. As a result, all astrophysical constraints [15] related to stellar cooling by majoron emission can easily be obeyed.

However, there is an important difference. In the seesaw majoron model, the smallness of neutrino masses is linked to the lepton number violation at a very high mass scale, i.e. $m_{\nu} \rightarrow 0$ as $\langle\sigma\rangle$ becomes large. In all the present models $m_{\nu} \rightarrow 0$ as $\langle\sigma\rangle \rightarrow 0$. The remarkable fact that no mass scale is introduced above the weak scale in any of these models is crucial in ensuring the importance of the invisible Higgs decay (1) relative to the standard modes such as $h \rightarrow f \bar{f}$. As we will see, the invisibly decaying Higgs boson signature may persist even in the limit where

\footnotetext{
$\ddagger$ For simplicity we assume that all of the Yukawa coupling constants are real in all that follows.
} 
$\langle\sigma\rangle \ll \mathcal{O}(1) \mathrm{TeV}$. This may be interesting from the point of view of processes such as neutrinoless double beta decay with majoron emission [13].

\subsection{Singlet majoron in " $\mu$-model"}

This is an $S U(2) \otimes U(1)$ variant [16] of the "seesaw" model with the heavy Dirac lepton suggested in ref. [17]. The relevant terms in the Lagrangian are

$$
h_{\nu} \ell^{T} C \nu^{c} H+M \nu^{c} C S+f \sigma S^{T} C S+h . c .
$$

They involve a bare Dirac mass term $M$ and the Yukawa couplings $h_{\nu}, f^{\S}$. These are described by arbitrary matrices in generation space. The first coupling generates the neutrino Dirac mass term $D=h_{\nu}\langle\phi\rangle$, while the third term gives rise to the Majorana mass term $\mu$ for the isosinglet $S$. This violates lepton number by two units, $\mu=f\langle\sigma\rangle$. The full mass matrix in two-component basis $\nu, \nu^{c}, S$ can be written as

$$
\left(\begin{array}{lll}
0 & D & 0 \\
D & 0 & M \\
0 & M & \mu
\end{array}\right) .
$$

For small values of the parameter $\mu \ll D \ll M$, the heavy leptons here are of quasiDirac type and the Majorana mass of the light, mostly isodoublet neutrino $\nu_{L}$ arises from the exchange of the heavy leptons, as indicated in Fig. 1(a). The resulting mass is given by

$$
m=\mu\left(\frac{D}{M}\right)^{2},
$$

Note the different relationship between $m_{\nu}$ and $\mu \lesssim\langle\sigma\rangle$, the lepton number breaking scale. This is a crucial feature of this model, which contrasts with the simplest seesaw model [11]. This difference arise from the fact that the the model contains a quasi-Dirac heavy lepton whose mass $\sim M$ is invariant under lepton number and is unrelated to neutrino masses. In contrast, the minimal seesaw model has a heavy Majorana lepton whose mass $\sim M$ is inversely related to that of the isodoublet

\footnotetext{
${ }^{\S} \mathrm{A} \nu^{c} \nu^{c} \sigma^{*}$ entry would not give a mass to the light neutrinos and can be forbidden by requiring supersymmetry.

』For simplicity we assume here only one generation. The complete form can be found in ref. $[16]$.
} 
neutrino. In both cases the heavy lepton admixture in the weak charged current is determined by the ratio $D / M$. However, in the " $\mu$-model" this value is restricted only by weak universality constraints, and not by limits on the neutrino masses [3]. The crucial point is that the heavy lepton mass here arises mostly from the entry $M$ which is invariant under lepton number, unlike the case of the seesaw model. As a result $M$ can be relatively low without implying too large $m_{\nu}$ values. In fact, in the limit where lepton number is exact neutrino masses are strictly forbidden [17].

As a result, there is a rich class of processes that can be enhanced for values of $D / M \lesssim 0.1$, well consistent with present weak universality constraints [14]. The resulting phenomenology has been considered in several papers. For masses below $m_{Z}$ the heavy quasi-Dirac leptons may be singly produced at LEP1, giving rise to striking events characterized by a large amount of missing energy [18]. For higher masses the existence of such neutral heavy leptons can at present only be probed through their indirect effects. For example, if we include mixing between the various generations we have the interesting possibility of flavour and/or CP violation [19], even in the limit where $\mu \rightarrow 0$, while the isodoublet neutrinos become strictly massless. As a result, processes such as $\mu \rightarrow e \gamma, \tau \rightarrow e \gamma, \mu-e$ conversion in nuclei, $Z \rightarrow e \tau$, etc. are not only allowed, but their rates are restricted only by the precision of weak universality tests. As a result, they can all be within the sensitivity of present experiments as well as of those expected at the upcoming $\tau$ factory [20].

\subsection{Singlet majoron in Zee-type models}

We now turn to models where neutrino masses are radiatively induced. The simplest possibility would be to consider models with just the three usual neutrinos. The prototype of these models was originally suggested by Zee [21]. Here we consider a variant which introduces the spontaneous violation of lepton number [22], so as to generate the majoron eq. (3). The relevant terms in the Lagrangian are the Yukawa couplings

$$
\frac{m_{i}}{\langle\phi\rangle} \bar{\ell}_{i} \phi e_{R i}+h_{i j} \bar{\ell}_{i} \tilde{\phi} e_{R i}+f_{i j} \ell_{i}^{T} C i \tau_{2} \ell_{j} \eta^{+}+\text {h.c. },
$$

where $i, j=e, \mu, \tau$. The first term is the canonical one, responsible for generating the charged lepton masses $m_{i}$ when the $S U(2) \otimes U(1)_{Y}$ gauge symmetry is broken by $\langle\phi\rangle$. The additional couplings involve another Higgs doublet $\tilde{\phi}$ as well as the Zee singlet; they are specified by matrices $f, g$ (in generation space), $f$ being antisymmetric. In 
addition we use the following quartic term in the scalar potential

$$
\lambda \phi \sigma_{2} \tilde{\phi} \eta^{+} \sigma+\text { h.c. }
$$

instead of the usual cubic term $\phi \sigma_{2} \tilde{\phi} \eta^{+}$that would explicitly violate lepton number. The quartic coupling fixes $L(\sigma)=2=-L(\eta)$ and induces a mixing between the physical singly charged scalars, which plays a crucial role in the radiative generation of neutrino mass, through the diagram in Fig. 1(b).

One can also consider the singlet majoron in a coloured version of the Zee model suggested in [13]. This is a variant of the previous model which is defined by the Lagrangian

$$
\frac{m_{i}}{\langle\phi\rangle} \bar{\ell}_{i} \phi e_{R i}+h_{i j} \ell_{i}^{T} C b_{L i j}^{c} \phi_{D}+f_{i j} \ell_{i}^{T} C Q_{L j} \eta_{S}+\lambda \phi \sigma_{2} \phi_{D} \eta_{S} \sigma+h . c .
$$

where $i, j=1 \ldots 3, \phi_{D}=\left(\begin{array}{c}\phi^{2 / 3} \\ \phi^{-1 / 3}\end{array}\right)$ and $\eta_{S}$ are colour triplet leptoquark scalar bosons; $\phi_{D}$ is an $S U(2) \otimes U(1)$ doublet with $Y=1 / 3$ and $L=-1$ while $\eta_{S}$ is a singlet with $Y=2 / 3$ and also $L=-1 ; b_{L}^{c}$ are $S U(2)$ singlet charged $1 / 3$ quarks. Again lepton number is spontaneously broken by $\langle\sigma\rangle$ and this generates the majoron, as in the previous cases. Neutrino masses are now induced from the second graph in Fig. $1(\mathrm{~b})$.

In this model the majoron-neutrino couplings are enhanced with respect to the first case of eq. (7), leading to the observability of neutrinoless double beta decay with majoron emission [13].

\subsection{Singlet majoron in models with sterile neutrinos}

Recently there has been a lot of interest in the possible existence of light sterile neutrinos [23, 24]. These models provide a common framework in terms of which to explain the solar neutrino data and the existence of a hot dark matter neutrino component in the universe, as recently suggested by COBE results on the large-scale structure of the universe [24]. They may also be relevant in connection with the atmospheric muon neutrino deficit as well as with the possible existence of anomalies in beta decays associated with heavy neutrinosll.

\footnotetext{
IIn fact they have been originally suggested in relation with the $17 \mathrm{keV} \beta$ decay anomaly.
} 
The simplest model [23] is again based on the $S U(2) \otimes U(1)$ gauge group, but extends the standard model by adding four singlet Higgs bosons $k^{++}, \eta^{+}, h^{+}$and $\sigma$ and one $S U(2) \otimes U(1)$ singlet neutrino $n u_{S}$. The Yukawa interactions are given by

$$
-\frac{\sqrt{2} m_{i}}{v} \bar{\ell}_{i} \phi e_{R i}+f_{i j} \ell_{i}^{T} C i \tau_{2} \ell_{j} h^{+}+h_{i j} e_{R i}^{T} C e_{R j} k^{++}+\xi_{i} \nu_{S}^{T} C e_{R i} \eta^{+}+\text {h.c. }
$$

where $\ell$ denotes a lepton doublet and $f, h, \xi$ are dimensionless Yukawa couplings. In addition the model contains the following crucial scalar self interactions

$$
\lambda_{k} \sigma h^{-2} k^{++}+\mu_{\eta} \sigma^{*} h^{-} \eta^{+}+\text {h.c. }
$$

where $\lambda_{k}$ is dimensionless and $\mu_{\eta}$ has dimensions of mass and its magnitude is at the weak scale. The model has a global lepton number symmetry $U(1)_{G}$ assigned canonically to the standard model states. The quantum number assignments and particle content are summarized in Table 1.

The neutrino mass matrix that follows from electroweak and $U(1)_{G}$ violation has the following form in the basis $\left(\nu_{e}, \nu_{\mu}, \nu_{\tau}, \nu_{S}\right)$

$$
M_{\nu}=\left(\begin{array}{cc}
m_{i j} & M_{i} \\
M_{j} & \mu
\end{array}\right) .
$$

Before $U(1)_{G}$ is broken the only non-zero entries are $M_{i}$. In this limit, two of the neutrinos are massless and the other two form a Dirac state $\nu_{D M}$ with mass

$$
m_{D M} \approx \sqrt{M_{e}^{2}+M_{\mu}^{2}+M_{\tau}^{2}}
$$

This mass is induced by the diagram in Fig. 2(b). This state is identified by two angles $\theta$ and $\varphi$ defined as

$$
\sin \theta=\frac{M_{e}}{m_{D M}} \quad \tan \varphi=\frac{M_{\mu}}{M_{\tau}} .
$$

Here

$$
M_{i}=\frac{\sum_{a} f_{i a} \xi_{a} m_{a}}{32 \pi^{2}} \sin 2 \beta \ln \left(\frac{M_{H_{2}}}{M_{H_{1}}}\right)^{2},
$$

where $\beta$ is the mixing angle of the scalar bosons and $M_{H_{i}}$ are their mass eigenvalues. For suitable choices of Yukawa couplings, the Dirac neutrino mass can be at the dark matter scale.

The entries $m_{i j}$ and $\mu$ only arise at the 2-loop level from the diagrams in Fig. 2(a) and 2(c). They are more highly suppressed also because they involve additional 
electroweak violating lepton mass insertions $m_{i j}$ or $U(1)_{G}$-violating $\langle\sigma\rangle$ insertions $\mu$. These are estimated as

$$
\begin{array}{r}
\mu \approx \frac{\lambda\langle\sigma\rangle \sum_{a, b} h_{a b} \xi_{a} \xi_{b}}{128 \pi^{4}} I_{\mu} \sin ^{2} 2 \beta \\
m_{i j} \approx-\frac{\lambda\langle\sigma\rangle \sum_{a, b} f_{i a} f_{j b} h_{a b} m_{a} m_{b}}{256 \pi^{4} M_{0}{ }^{2}} I_{m},
\end{array}
$$

where $M_{0}$ is a typical Higgs boson mass, $I_{m}$ and $I_{\mu}$ are the relevant Feynman integrals, typically of order one $\left(I_{\mu} \rightarrow 0\right.$, when $\left.M_{H_{1}} \rightarrow M_{H_{2}}\right)$. These terms give masses to the lowest-lying neutrinos responsible for the explanation of the solar neutrino data. For suitable values of the parameters, these are in the right range to give a solution of the solar neutrino deficit via matter enhanced $\nu_{e}$ to $\nu_{\mu}$ transitions. These MSW transitions involve an additional angle needed to diagonalize the resulting light mass matrix. For alternative choices of parameters, there can also be an explanation of the muon deficit in atmospheric neutrinos [24].

\section{Scalar Higgs potential}

To complete the specification of the models, we must now discuss the associated scalar potential. It is clear that all these models are characterized by two basic types of scalar potentials, when one considers only the neutral sector needed to determine the vacuum. The models in sections 2.1 and 2.3 are characterized by a scalar potential with one doublet and one singlet Higgs multiplet, while the scalar potential of the remaining models contains an additional Higgs doublet. We discuss both of these in turn.

\subsection{One scalar Higgs doublet and one singlet}

This case was discussed in [10]. We recall the basic aspects. The scalar potential is given by

$$
V_{N_{1}}=\mu_{\phi}^{2} \phi^{\dagger} \phi+\mu_{\sigma}^{2} \sigma^{\dagger} \sigma+\lambda_{1}\left(\phi^{\dagger} \phi\right)^{2}+\lambda_{2}\left(\sigma^{\dagger} \sigma\right)^{2}+\delta\left(\phi^{\dagger} \phi\right)\left(\sigma^{\dagger} \sigma\right)
$$

Terms like $\sigma^{2}$ are omitted above in view of the imposed $U(1)$ invariance under which we require $\sigma$ to transform non-trivially and $\phi$ to be trivial. Let $\sigma \equiv \frac{w}{\sqrt{2}}+\frac{R_{2}+i I_{2}}{\sqrt{2}}$, $\phi^{0} \equiv \frac{v}{\sqrt{2}}+\frac{R_{1}+i I_{1}}{\sqrt{2}}$, where we have set $\langle\sigma\rangle=\frac{w}{\sqrt{2}}$ and $\left\langle\phi^{0}\right\rangle=\frac{v}{\sqrt{2}}$. The above potential 
then leads to a physical massless Goldstone boson, namely the majoron $J \equiv \operatorname{Im} \sigma$ and two massive neutral scalars $H_{i}(i=1,2)$ :

$$
H_{i}=\hat{O}_{i j} R_{j}
$$

The mixing $\hat{O}$ can be parametrized as

$$
\hat{O}=\left(\begin{array}{cc}
\cos \theta & \sin \theta \\
-\sin \theta & \cos \theta
\end{array}\right)
$$

The mixing angle $\theta$ as well as the Higgs masses $M_{i}^{2}$ are related to the parameters of the potential in the following way:

$$
\begin{aligned}
2 \delta v w & =\left(M_{2}^{2}-M_{1}^{2}\right) \sin 2 \theta, \\
2 \lambda_{1} v^{2} & =M_{1}^{2} \cos ^{2} \theta+M_{2}^{2} \sin ^{2} \theta, \\
2 \lambda_{2} w^{2} & =M_{2}^{2} \cos ^{2} \theta+M_{1}^{2} \sin ^{2} \theta . \\
\tan 2 \theta & =-\frac{\delta v \omega}{\lambda_{1} v^{2}-\lambda_{2} \omega^{2}} .
\end{aligned}
$$

The masses $M_{1,2}^{2}$, the mixing angle $\theta$, and the ratio of two vacuum expectation values $\tan \beta=\frac{v}{w}$ can be taken as independent parameters in terms of which all couplings can be fixed. There are no physical charged Higgs bosons in this case.

The potential in eq. (18) generates the following coupling of $H_{i}$ to the majoron $J$ :

$$
\mathcal{L}_{J}=\frac{\left(\sqrt{2} G_{F}\right)^{1 / 2}}{2} \tan \beta\left[M_{2}^{2} \cos \theta H_{2}-M_{1}^{2} \sin \theta H_{1}\right] J^{2}
$$

\subsection{Two scalar Higgs doublets and one singlet}

The part of the scalar potential containing the neutral Higgs fields is given in this case by

$$
\begin{aligned}
V_{N 2}= & \mu_{i}^{2} \phi_{i}^{\dagger} \phi_{i}+\mu_{\sigma}^{2} \sigma^{\dagger} \sigma+\lambda_{i}\left(\phi_{i}^{\dagger} \phi_{i}\right)^{2}+\lambda_{\sigma}\left(\sigma^{\dagger} \sigma\right)^{2} \\
& +\lambda_{12}\left(\phi_{1}^{\dagger} \phi_{1}\right)\left(\phi_{2}^{\dagger} \phi_{2}\right)+\lambda_{13}\left(\phi_{1}^{\dagger} \phi_{1}\right)\left(\sigma^{\dagger} \sigma\right)+\lambda_{23}\left(\phi_{2}^{\dagger} \phi_{2}\right)\left(\sigma^{\dagger} \sigma\right) \\
& +\delta\left(\phi_{1}^{\dagger} \phi_{2}\right)\left(\phi_{2}^{\dagger} \phi_{1}\right)+\frac{1}{2} \beta\left[\left(\phi_{1}^{\dagger} \phi_{2}\right)^{2}+\text { h.c. }\right],
\end{aligned}
$$

where a sum over repeated indices $i=1,2$ is assumed. Here $\phi_{1,2}$ are the doublet fields and $\sigma$ corresponds to the singlet carrying non-zero lepton number. 
In writing down the above equation, we have imposed a discrete symmetry $\phi_{2} \rightarrow-\phi_{2}$ needed to obtain natural flavour conservation in the presence of more than one Higgs doublets. For simplicity, we assume all couplings and VEVs to be real. Then the conditions for the minimization of the above potential are easy to work out and are given by

$$
\begin{gathered}
\mu_{1}^{2}+v_{1}^{2} \lambda_{1}+\frac{1}{2}\left(\lambda_{12}+\delta\right) v_{2}^{2}+\frac{1}{2} \lambda_{13} v_{3}^{2}+\frac{1}{2} \beta v_{2}^{2}=0 \\
\mu_{2}^{2}+v_{2}^{2} \lambda_{2}+\frac{1}{2}\left(\lambda_{12}+\delta\right) v_{1}^{2}+\frac{1}{2} \lambda_{23} v_{3}^{2}+\frac{1}{2} \beta v_{1}^{2}=0 \\
\mu_{3}^{2}+v_{3}^{2} \lambda_{3}+\frac{1}{2} \lambda_{13} v_{1}^{2}+\frac{1}{2} \lambda_{23} v_{2}^{2}=0 .
\end{gathered}
$$

These conditions can be used to work out the mass matrix for the Higgs fields. To this end we shift the fields as $(i=1,2)$ :

$$
\begin{aligned}
& \phi_{i}=\frac{v_{i}}{\sqrt{2}}+\frac{R_{i}+i I_{i}}{\sqrt{2}}, \\
& \sigma=\frac{\omega}{\sqrt{2}}+\frac{R_{3}+i I_{3}}{\sqrt{2}} .
\end{aligned}
$$

The masses of the CP-even fields $R_{a}(a=1 \ldots 3)$ are obtained from

$$
\mathcal{L}_{\text {mass }}=\frac{1}{2} R^{T} M_{R}^{2} R
$$

with

$$
M_{R}^{2}=\left(\begin{array}{ccc}
2 \lambda_{1} v_{1}^{2} & \left(\beta+\lambda_{12}+\delta\right) v_{1} v_{2} & \lambda_{13} v_{1} v_{3} \\
\left(\beta+\lambda_{12}+\delta\right) v_{1} v_{2} & 2 \lambda_{2} v_{2}^{2} & \lambda_{23} v_{2} v_{3} \\
\lambda_{13} v_{1} v_{3} & \lambda_{23} v_{2} v_{3} & 2 \lambda_{3} v_{3}^{2}
\end{array}\right) .
$$

The physical mass eigenstates $H_{a}$ are related to the corresponding weak eigenstates as

$$
H_{a}=O_{a b} R_{b}
$$

where $O$ is a $3 \times 3$ matrix diagonalizing $M_{R}^{2}$

$$
O M_{R}^{2} O^{T}=\operatorname{diag}\left(M_{1}^{2}, M_{2}^{2}, M_{3}^{2}\right)
$$

The majoron is given in this case by $J=I_{3}$. The coupling of the physical Higgses to $J$ follows from eq. (23). As in the previous case, it is possible to express this 
coupling entirely in terms of the masses $M_{a}^{2}$ and the mixing angles characterizing the matrix $O$

$$
\begin{aligned}
\mathcal{L}_{J} & =\frac{1}{2} J^{2}\left(2 \lambda_{3} v_{3} R_{3}+\lambda_{13} v_{1} R_{1}+\lambda_{23} v_{2} v_{3} R_{2}\right), \\
& =\frac{J^{2}}{2 v_{3}}\left(M_{R}^{2}\right)_{3 a} R_{a}, \\
& =\frac{1}{2}\left(\sqrt{2} G_{F}\right)^{1 / 2} \tan \gamma\left(O^{T}\right)_{3 a} M_{a}^{2} H_{a} J^{2} .
\end{aligned}
$$

$\tan \gamma \equiv \frac{V}{v_{3}} ; V=\left(v_{1}^{2}+v_{2}^{2}\right)^{1 / 2}$. We have made use of eq. (31) and eq. (32) in writing the last line.

Unlike in the previous case, there now exists also a massive CP-odd state $A$, related to the doublet fields as follows:

$$
A=\frac{1}{V}\left(v_{2} I_{1}-v_{1} I_{2}\right)
$$

Its mass is given by

$$
M_{A}^{2}=-\beta V^{2}
$$

When $\beta \rightarrow 0$ this pseudoscalar boson becomes massless, as the potential acquires a new symmetry.

\section{Higgs production and decay}

The Higgs can be produced at the $e^{+} e^{-}$collider through its couplings to $Z$. Although the $S U(2) \otimes U(1)$ singlet field $\sigma$ does not couple to $Z$, all of the CP-even mass eigenstates $H$ introduced in the last section have couplings to the $Z$ through mixing. The couplings relevant for their production through the Bjorken process are given as follows $(a=1 \ldots 3)$

$$
\mathcal{L}_{H Z Z}=\left(\sqrt{2} G_{F}\right)^{1 / 2} M_{Z}^{2} Z_{\mu} Z^{\mu}\left[\frac{v_{1}}{V} O_{1 a}+\frac{v_{2}}{V} O_{2 a}\right] H_{a}
$$

in the two doublet case and $(i=1,2)$

$$
\mathcal{L}_{H Z Z}=\left(\sqrt{2} G_{F}\right)^{1 / 2} M_{Z}^{2} Z_{\mu} Z^{\mu} \hat{O}_{i 1} H_{i}
$$

for the case considered in section 3.1. As long as the mixing appearing in eq. (39) and eq. (38) are $\mathcal{O}(1)$, all Higgs bosons can have significant couplings and hence appreciable production rates through the Bjorken process. 
In case of the two doublet model, the $H_{a}$ can also be produced in association with the CP-odd field $A$ through the coupling

$$
\mathcal{L}_{H A Z}=-\frac{g}{\cos \theta_{W}} Z^{\mu}\left[\frac{v_{2}}{V} O_{1 a}-\frac{v_{1}}{V} O_{2 a}\right] H_{a} \overleftrightarrow{\partial^{\mu}} A
$$

The width for the invisible $H$ decay can be parametrized by

$$
\Gamma(H \rightarrow J J)=\frac{\sqrt{2} G_{F}}{32 \pi} M_{H}^{3} g_{H J J}^{2} .
$$

The one doublet model contains two Higgses $H_{i}$ whose couplings are given by

$$
g_{H_{i} J J}=\tan \beta \quad \hat{O}_{i 2}
$$

The analogous couplings in the case of the model of section 3.2 are given by $(a=$ $1 \ldots 3)^{* *}$

$$
g_{H_{a} J J}=\tan \gamma \quad O_{a 3}
$$

The rate for $H \rightarrow b \bar{b}$ also gets diluted in comparison to the standard model prediction, because of the mixing effects. Explicitly one has

$$
\Gamma(H \rightarrow b \bar{b})=\frac{3 \sqrt{2} G_{F}}{8 \pi} M_{H} m_{b}^{2}\left(1-4 m_{b}^{2} / M_{H}^{2}\right)^{3 / 2} g_{H b \bar{b}}^{2},
$$

with

$$
\begin{array}{ll}
g_{H_{i} b \bar{b}}=\hat{O}_{i 1} & \text { (one-doublet model) } \\
g_{H_{a} b \bar{b}}=O_{a 1} & \text { (two-doublet model) }
\end{array}
$$

The last coupling depends upon how the charged $-1 / 3$ quarks transform under the symmetry which avoids the flavour changing neutral currents in the presence of the two Higgs doublets. We have assumed that this symmetry allows only the $\phi_{1}$ to couple to the $d$-type quarks.

The width of the Higgs decay to the $J J$ relative to the conventional $b \bar{b}$ mode depends upon the mixing angles. The invisible mode is expected to dominate if the lepton number is broken around or below the weak scale. In order to appreciate this point, let us consider the relatively simple situation [10] with only one Higgs doublet, as in the model of section 3.1. One could imagine three cases: (i) $\omega \approx v, \quad$ (ii) $\omega \gg v$ and (iii) $\omega \ll v$. It follows from eq. (21) that in the first case, the mixing among the doublet and singlet field will be $\mathcal{O}(1)$ if the parameters of the quartic terms in the

\footnotetext{
** Additional contributions due to the decay of $H_{a}$ to $A A$ may exist.
} 
Higgs potential are similar in magnitude. As a result, the production as well as the decay of both physical Higgs bosons $H_{i}$ will be comparable and could be observable. The relative branching ratio in this case is given by

$$
\begin{aligned}
\frac{\Gamma\left(H_{1} \rightarrow J J\right)}{\Gamma\left(H_{1} \rightarrow b \bar{b}\right)} & =\frac{1}{12}\left(\frac{M_{1}}{m_{b}}\right)^{2}\left(1-4 m_{b}^{2} / M_{1}^{2}\right)^{-3 / 2}(\tan \beta \tan \theta)^{2} \\
& \approx 8\left(\frac{M_{1}}{50 \mathrm{GeV}}\right)^{2}(\tan \beta \tan \theta)^{2}
\end{aligned}
$$

A similar expression with $\tan \theta$ replaced by $\cot \theta$ holds in the case of $H_{2}$. It is clear that a Higgs boson with $M_{H}>50 \mathrm{GeV}$ decays mostly invisibly if $\tan \beta$ and $\tan \theta$ are $\mathcal{O}(1)$. The production of $H_{1}\left(H_{2}\right)$ gets diluted compared to the standard model prediction by $\cos ^{2} \theta\left(\sin ^{2} \theta\right)$. If $\omega$ and $v$ are very different from each other then the mixing angle in eq. (21) is very small. Hence in cases (ii) and (iii), only the predominantly doublet component $\left(H_{1}\right)$ will be produced. Use of eq. $(21)$ in the basic majoron coupling, eq. (22), reveals that

$$
\begin{gathered}
\lim _{\omega \gg v} \mathcal{L}_{J}=\left(\sqrt{G_{F}}\right)^{1 / 2} v \omega\left(\lambda_{2} H_{2}+H_{1} O\left(\frac{v^{3}}{\omega^{3}}\right)\right) J^{2}, \\
\lim _{\omega \ll v} \mathcal{L}_{J}=\frac{1}{2}\left(\sqrt{2} G_{F}\right)^{1 / 2} v^{2}\left(\delta H_{1}+H_{2} O\left(\frac{\omega}{v}\right)\right) J^{2} .
\end{gathered}
$$

It follows that if $\omega \gg v$ then only the singlet field decays to two majorons. But this cannot be produced. In the converse case, $\omega \ll v$, the doublet field mainly ${ }^{\dagger \dagger}$ decays to majorons and this also gets produced without any substantial suppression relative to the standard model predictions, in view of its small mixing with the singlet component. This case is then the ideal from the point of view of the observability of the invisible decay and may also lead to the observability of neutrino-majoron couplings and to that of neutrinoless double beta decay with majoron emission [13]. As would be expected, the presence of one more doublet does not qualitatively change this conclusion. This is demonstrated in the appendix.

\section{Discussion}

The Higgs can decay invisibly in a wide class of $S U(2) \otimes U(1)$ singlet majoron models. We have shown that the production of such Higgs bosons as well as their invisible decay width could be sizeable. In fact the Higgs decay width arising from eq. (1)

${ }^{\dagger \dagger}$ The factor $(\tan \beta \tan \theta)^{2}$ appearing in eq. (46) goes to a constant value $\frac{\delta^{2}}{4 \lambda_{1}^{2}}$ in this limit. 
can dominate over that of the standard $b \bar{b}$ mode in all such models. This leads to events with large missing energy carried by the majoron pair. Since this signature is very different from the conventional Higgs decay, a reanalysis of the present Higgs search strategies is needed. A comparison of some of the existing LEP data with the one-doublet model of section (3.1) has already been [10] given, treating $\sin \theta$ and $\tan \beta$ as independent parameters. Use of more data and a similar comparison of the two doublet model is worth while. While this is not the aim of the present work, we will make some comments on the main phenomenological implications.

The search strategy for the Higgs depends upon its mass and on the decay characteristics relevant for this mass. Accordingly, a Higgs boson with $M_{H} \leq 80$ $\mathrm{GeV}$ can be looked for at LEP1 or LEP2. Heavier Higgs bosons can be searched at hadron colliders. If $M_{H}$ is less than twice the mass of the $W$, one has to rely upon its rare decays such as $\gamma \gamma$, while a heavier Higgs boson can be found through its $W W$ and $Z Z$ decay modes. All of these searches can be substantially affected once the Higgs decay to majorons becomes possible. Consider, for example, the searches being carried out at LEP for Higgs with masses greater than about $10 \mathrm{GeV}$. This depends upon detecting the Higgs decay to the $b \bar{b}$ pair. Since the branching ratio for this mode gets diluted in the presence of the invisible decay, the Higgs could have escaped detection at LEP. This was analysed in ref. [10]. It was shown that a large region in the parameter space still remains unconstrained in the simple model of section 3.1. In particular, any value of $M_{H}$ is allowed for a suitable range in $\theta$ and $\tan \beta$. The models with two Higgs doublets (section 2.2) are even less constrained owing to the presence of more parameters. But in this case there exists an additional way to produce the Higgs, namely through the associated process $Z \rightarrow A H$, eq. (40). In the general two Higgs doublet models and in the supersymmetric ones, the processes $Z \rightarrow Z^{*} H$ and $Z \rightarrow A H$ are known to provide complementary information on the relevant mixing parameters. In the present case, since there exists an additional mixing involving the singlet field $\sigma$, the mixings appearing in eq. (38) and eq. (40) are not related in a simple way, as in the two doublet case. However, if the lepton number breaking does occur at very low scale then the mixing between doublet and singlet is very small (see appendix) and the above production mechanisms can be used simultaneously in order to restrict the parameters of the model.

Invisible Higgs decay could be directly observed if the Higgs boson is produced in association with a photon, $W$ or $Z$. The latter can be used as a tag of the 
invisible mode. The production of the Higgs in association with the photon at LEP is unfortunately quite suppressed. But the other decays could in principle be used. The possibility of invisible Higgs decays is specially interesting for the case of hadron colliders [14]. In fact, a recent analysis has studied the feasibility of detecting an invisible Higgs produced in association with $W, Z$ at hadron colliders [25]. They conclude that, with reasonable assumptions, a dominantly decaying Higgs boson lighter than $2 m_{Z}$ can be detected at both LHC and SSC. It is interesting to note that this intermediate Higgs mass region is one where the traditional tree level $f \bar{f}$ mode is not useful.

The main conclusion of the present work, which we would like to stress again, is that the invisible Higgs decays are a generic feature of a wide class of singlet majoron models of neutrino mass generation, which are interesting in their own right. Since the majoron does not appreciably couple to fermions nor to gauge bosons, such invisible decay may be a very good way to test the validity of the majoron hypothesis itself. Moreover, since such decay could hide the Higgs, special efforts would be needed to look for the Higgs boson in this case. But the Higgs boson, if discovered through its invisible decay, would tell us that not only $S U(2) \otimes U(1)$ but also the lepton number is a spontaneously broken symmetry.

This work was partially supported by CICYT (Spain). We thank Riccardo Barbieri for useful discussions. A.S.J. thanks S. Rindani for discussions related to this work. 


\section{Appendix}

We discuss here the case of very small violation of lepton number symmetry. Specifically, we shall assume $v_{3} \ll v_{1,2}$. The mass matrix $M_{R}^{2}$ for the CP-even fields can be written in the $\left(R_{1}, R_{2}, R_{3}\right)$ basis as

$$
M_{R}^{2}=V^{2}\left(\begin{array}{ccc}
A & B & \epsilon_{1} \\
B & C & \epsilon_{2} \\
\epsilon_{1} & \epsilon_{2} & \epsilon_{3}^{2}
\end{array}\right),
$$

where,

$$
\epsilon_{1} \equiv \lambda_{13} \frac{v_{1} v_{3}}{V^{2}} ; \epsilon_{2} \equiv \lambda_{23} \frac{v_{2} v_{3}}{V^{2}} ; \epsilon_{3}^{2}=2 \frac{\lambda_{3} v_{3}^{2}}{V^{2}} .
$$

The parameters $A, B, C$ can be read from eq. (30) and are $\mathcal{O}(1)$, while $\epsilon_{1,2,3}$ are similar in magnitude but are much smaller than $A, B, C$. The matrix $M_{R}^{2}$ can be approximately diagonalized by the $O$ given by

$$
O=R_{13}\left(\theta_{3}\right) R_{23}\left(\theta_{2}\right) R_{12}\left(\theta_{1}\right)
$$

where $R_{a b}$ denotes a rotation in the $a b$ plane. To leading order, the masses and mixing angles are given by

$$
\begin{aligned}
& \tan 2 \theta_{1} \approx \frac{2 B}{C-A}, \\
& \tan 2 \theta_{2} \approx-\frac{2\left(\sin \theta_{1} \epsilon_{1}+\cos \theta_{1} \epsilon_{2}\right)}{M_{2}^{2}}, \\
& \tan 2 \theta_{3} \approx-\frac{2 \cos \theta_{2}\left(-\sin \theta_{1} \epsilon_{2}+\cos \theta_{1} \epsilon_{1}\right)}{M_{1}^{2}}, \\
& M_{1}^{2} \approx A \cos ^{2} \theta_{1}+C \sin ^{2} \theta_{1}-\sin 2 \theta_{1} B \\
& M_{2}^{2} \approx A \sin ^{2} \theta_{1}+C \cos ^{2} \theta_{1}+\sin 2 \theta_{1} B .
\end{aligned}
$$

The other eigenvalue $M_{3}^{2}$ is of $\mathcal{O}\left(\epsilon_{3}^{2}\right)$. The elements of the mixing matrix relevant to determine the Higgs coupling to a majoron [eq. (33)] can be read off from eq. (51) and eq. (52).

$$
\begin{aligned}
& O_{13} \approx \frac{\cos ^{2} \theta_{2}\left(-\sin ^{2} \theta_{1} \epsilon_{2}+\cos ^{2} \theta_{1} \epsilon_{1}\right)}{M_{1}^{2}} \equiv \frac{\kappa_{1} v_{3} v_{1}}{M_{1}^{2}}, \\
& O_{23} \approx \frac{\sin \theta_{1} \epsilon_{1}+\cos \theta_{1} \epsilon_{2}}{M_{2}^{2}} \equiv \frac{\kappa_{2} v_{2} v_{3}}{M_{2}^{2}} .
\end{aligned}
$$


The parameters $\kappa_{1,2}$ are $\mathcal{O}(1)$. Using eq. (33) and eq. (54), it is seen that in the limit $\epsilon_{1,2,3} \ll 1$, the majoron mainly couples to the predominantly doublet fields as follows:

$$
\mathcal{L}_{J} \approx \frac{1}{2}\left[\kappa_{1} v_{1} H_{1}+\kappa_{2} v_{2} H_{2}\right] J^{2} .
$$

The coupling of $Z$ to $H_{1,2}$ and $A$, eq. (33) and eq. (54), reduces in this case to

$$
\mathcal{L}_{H A Z} \approx-\frac{g}{\cos \theta_{W}} Z^{\mu}\left[\sin \left(\theta_{1}+\eta\right) H_{1}-\cos \left(\theta_{1}+\eta\right) H_{2}\right] \overleftrightarrow{\partial^{\mu}} A
$$

$\tan \eta=\frac{v_{2}}{v_{1}}$. Likewise, the $H Z Z$ coupling of eq. (38) reduces to

$$
\mathcal{L}_{H Z Z} \approx\left(\sqrt{2} G_{F}\right)^{1 / 2} M_{Z}^{2} Z_{\mu} Z^{\mu}\left[\cos \left(\theta_{1}+\eta\right) H_{1}+\sin \left(\theta_{1}+\eta\right) H_{2}\right]
$$

This is similar to the conventional couplings in the two doublet case. 


\section{Figure captions}

\section{Figure 1}

Diagrams in 1(a) and 1(b) generate non-zero neutrino masses in the models of sections 2.1 and 2.2, respectively.

\section{Figure 2}

The diagram in 2(b) generates the Dirac neutrino mass in the model of section 2.3, while those in 2(a) and 2(c) give the small Majorana entries $m_{i j}$ and $\mu$ that can be $\mathcal{O}\left(10^{-3}\right) \mathrm{eV}$. 


\section{References}

[1] P. W. Higgs, Phys. Lett. 12, 132 (1964).

[2] D. Decamp et al. (ALEPH Collaboration), Phys. Lett. B236, 233 (1990), B237, 291 (1990), B241, 141 (1990), B245, 289 (1990), B246, 306 (1990); preprint CERN-PPE/91-149; P. Abreu et al. (DELPHI Collaboration), Nucl. Phys. B342, 1 (1990); Z. Phys. C51, 25 (1991); and preprint CERN-PPE/91-132; B. Adeva et al. (L3 Collaboration), Phys. Lett. B283, 454 (1992) and references therein; M.Z. Akrawy et al. (OPAL Collaboration), Phys. Lett. B236, 224 (1990), B251, 211 (1990) and B253, 511 (1991); P. D. Acton et. al. Phys. Lett. B268, 122 (1991).

[3] J. W. F. Valle, Prog. Part. Nucl. Phys. 26, 91 (1991) and references therein.

[4] R. E. Scrock and M. Suzuki, Phys. Lett. 10B, 250 (1982); L. F. Li, Y. Liu and L. Wolfenstein, Phys. Lett. B159, 45 (1985). See also D. Chang and W. Keung, Phys. Lett. B217, 238 (1989).

[5] G. Gelmini and M. Roncadelli, Phys. Lett. B99, 411 (1981).

[6] J. Steinberger, in Electroweak Physics Beyond the Standard Model, ed. J. W. F. Valle and J. Velasco (World Scientific, Singapore, 1992), p. 3.

[7] J. D. Bjorken, Stanford preprint SLAC-PUB-5673 (1991). R. Barbieri and L. Hall, Nucl. Phys. B364, 27 (1991).

[8] J. C. Romao, F. de Campos and J. W. F. Valle, CERN preprint CERNTH.6586/92 (1992), Phys. Lett. B, in press.

[9] A. Masiero and J. W. F. Valle, Phys. Lett. B251, 273 (1990). J. C. Romao, C. A. Santos and J. W. F. Valle, Phys. Lett. B288, 311 (1992).

[10] A. S. Joshipura and S. Rindani, ICTP Report IC/92/193 (1992).

[11] Y. Chikashige, R. N. Mohapatra and R. D. Peccei, Phys. Lett. 98B, 265 (1980).

[12] E. D. Carlson and L. B. Hall, Phys. Rev. D40, 3187 (1985); G. Jungman and M. Luty, Nucl. Phys. B361, 24 (1991). 
[13] Z. Berezhiani, A. Smirnov and J. W. F. Valle, Valencia preprint FTUV/92-20 (1992), Phys. Lett. B, in press.

[14] J. W. F. Valle, CERN -TH. 6626/92, Physics at New Accelerators: Looking Beyond the Standard Model, invited talk at the XV Int. Conference on Neutrino Physics and Astrophysics, ed. A. Morales et al (North-Holland, Amsterdam 1992).

[15] J. E. Kim, Phys. Rep. 150, 1 (1987), and references therein.

[16] M. C. Gonzalez-Garcia and J. W. F. Valle, Phys. Lett. B216, 360 (1989).

[17] R. Mohapatra and J. W. F. Valle, Phys. Rev. D34, 1642 (1986).

[18] M. Dittmar, M. C. Gonzalez-Garcia, A. Santamaria and J. W. F. Valle, Nucl. Phys. B332, 1 (1990). M. C. Gonzalez-Garcia, A. Santamaria, and J. W. F. Valle, Nucl. Phys. B342, 108 (1990).

[19] J. Bernabéu, A. Santamaria, J. Vidal, A. Mendez and J. W. F. Valle, Phys. Lett. B187, 303 (1987). G. C. Branco, M. N. Rebelo and J. W. F. Valle, Phys. Lett. B225, 385 (1989). N. Rius and J. W. F. Valle, Phys. Lett. 246B, 249 $(1990)$.

[20] M. C. Gonzalez-Garcia and J. W. F. Valle, Mod. Phys. Lett. A7, 477 (1992).

[21] A. Zee, Phys. Lett. B93, 389 (1980).

[22] R. Barbieri and L. Hall, Nucl. Phys. B364, 27 (1991). Z. Berezhiani, unpublished.

[23] L. Bento and J. W. F. Valle, Phys. Lett. B264, 373 (1991).

[24] J. Peltoniemi, D. Tommasini and J. W. F. Valle, CERN-TH.6624/9D

[25] S. Frederiksen, N. Johnson, G. Kane and J. Reid, SSCL-preprint-577 (1992); J. C. Romao, F. de Campos, L. Diaz-Cruz and J. W. F. Valle, Valencia preprint FTUV/92-39 (1992). 
Table 1: $S U(2) \times U(1)_{Y}$ and lepton number assignments of the leptons and Higgs scalars. Quarks are $U(1)_{L}$ singlets.

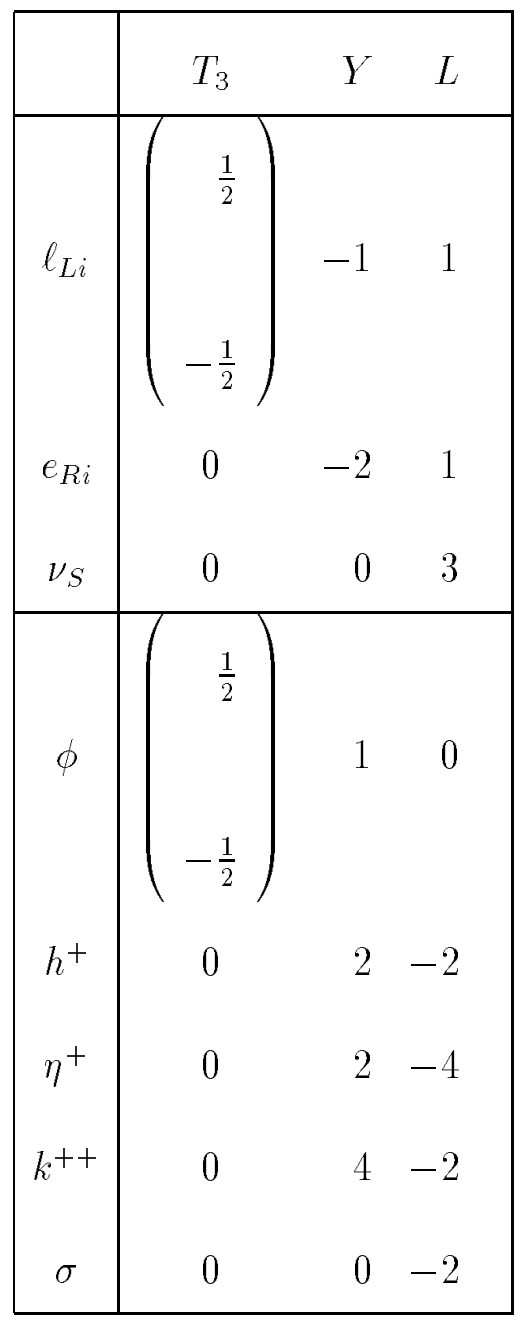




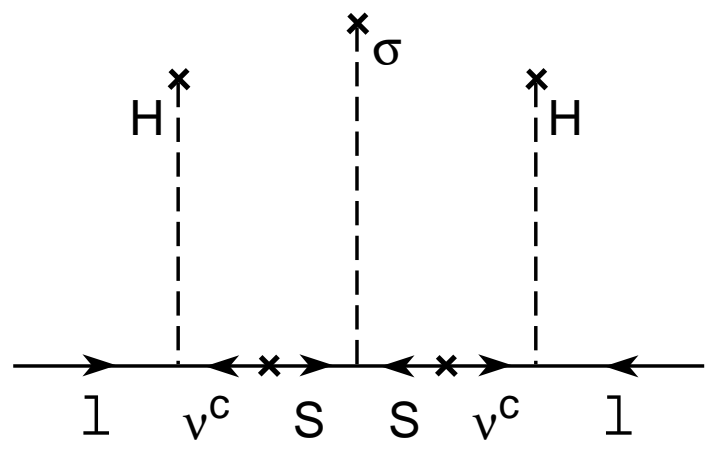

Fig. 1a
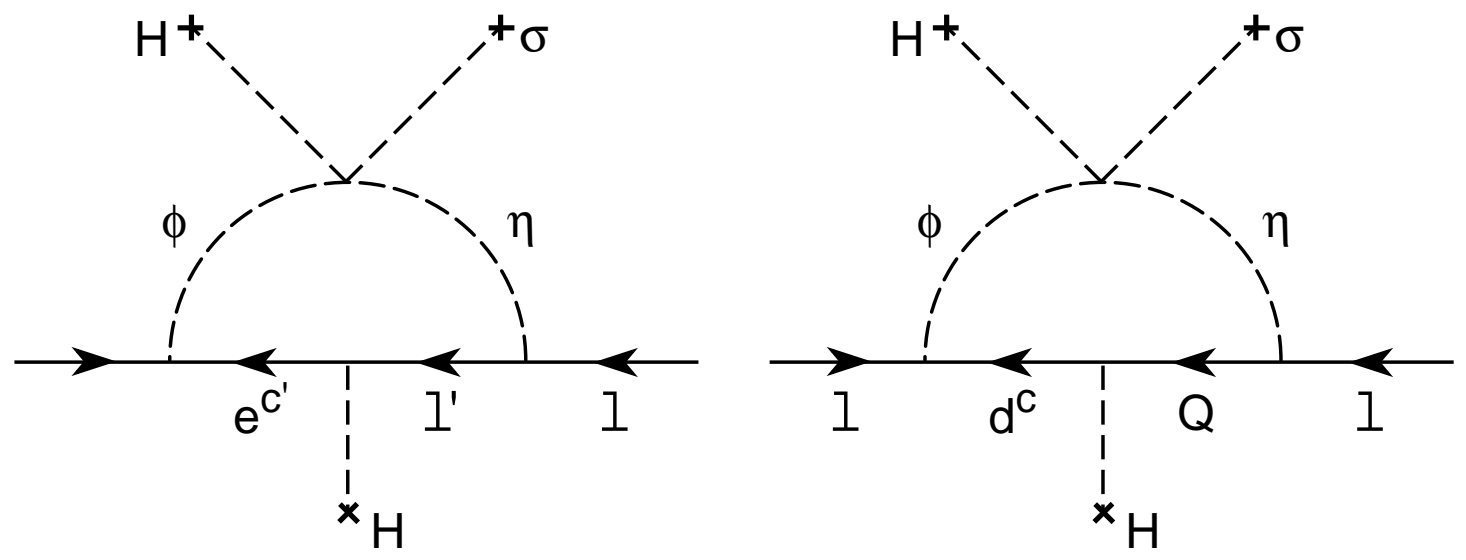

Fig. 1b 


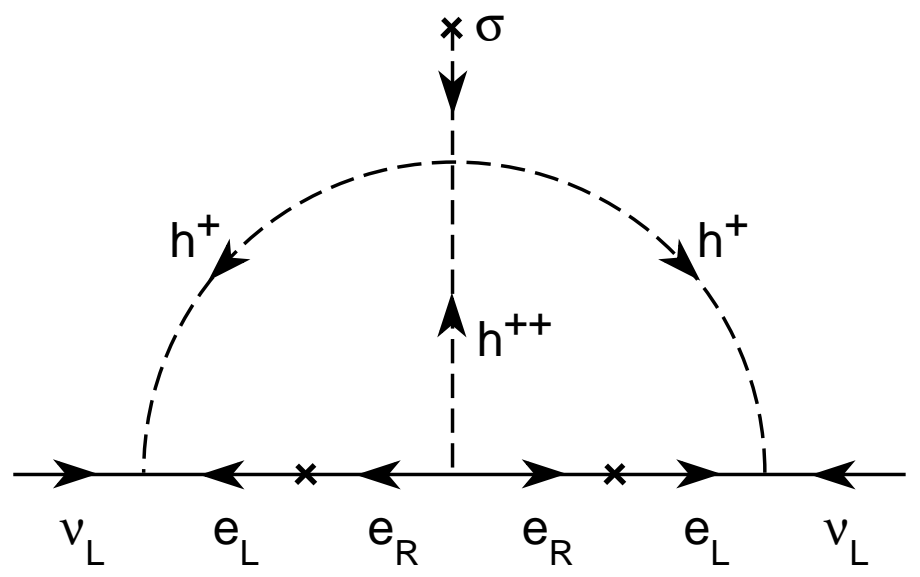

(a)

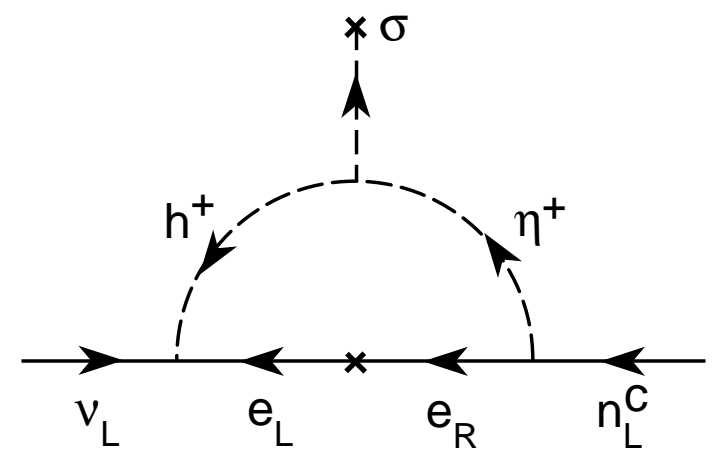

(b)

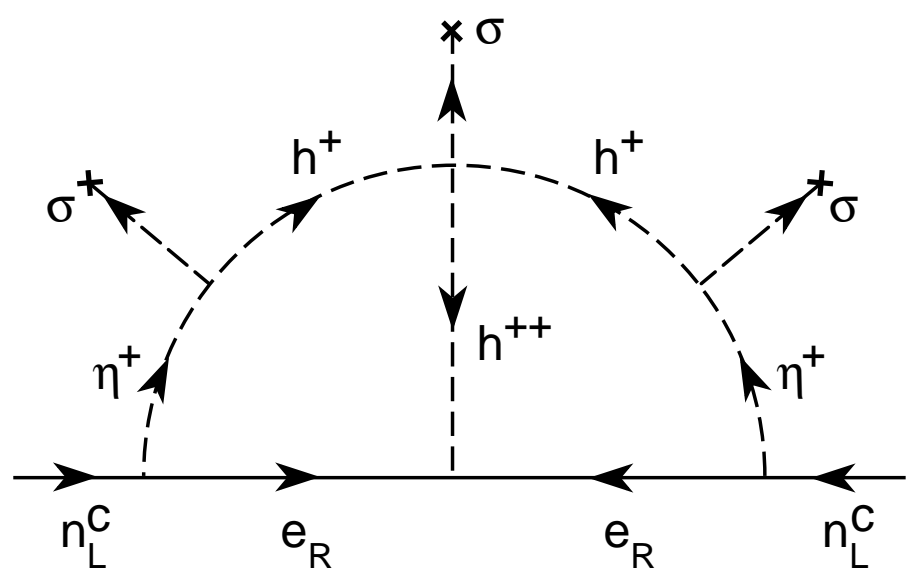

(c)

Fig. 2 